\title{
UM DIAGNÓSTICO SOBRE A PRÁTICA DE ATIVIDADES FÍSICAS POR PESSOAS COM DEFICIÊNCIA VISUAL
}

Rafaella Righes Machado, Universidade Federal de Santa Maria - UFSM, Santa Maria, Rio Grande do Sul - Brasil

Luciana Erina Palma, Universidade Federal de Santa Maria - UFSM, Santa Maria, Rio Grande do Sul - Brasil

Sofia Wolker Manta, Universidade Federal de Santa Catarina - UFSC, Florianópolis, Santa Catarina - Brasil

Greice Rosso Lehnhar, Universidade Federal de Santa Maria - UFSM, Santa Maria, Rio Grande do Sul - Brasil

\section{RESUMO}

O objetivo deste estudo foi verificar as Instituições Especializadas de Ensino, Escolas Públicas Regulares e Associações da cidade de Santa Maria/RS que possuem alunos com deficiência visual e diagnosticar a prática de atividades físicas por esta população. Para a coleta de dados foi utilizada uma Ficha de Informações. Verificou-se, 01 Associação, 02 Escolas Estaduais e 09 Escolas Municipais, perfazendo um total de 65 participantes com deficiência visual. Prevaleceram maiores percentuais de não participação em AF por estes indivíduos, sendo que as atividades realizadas, em sua maioria eram em aulas de Educação Física. Conclui-se que há poucas Instituições de Ensino frequentadas por pessoas com deficiência visual, bem como insuficiente o atendimento na área de atividades físicas a esta população.

Palavras-Chave: Pessoas com deficiência visual; Atividade física; Instituições de ensino.

\section{A DIAGNOSIS OF PHYSICAL ACTIVITY FOR PEOPLE WITH VISUAL DISABILITIES}

\begin{abstract}
The aim of this study was to verify the Specialized Education Institutions, Associations and Regular Public Schools in the city of Santa Maria / RS that have visually impaired students and diagnose the physical activity for this population. To collect one data sheet information was used. There was 01 Association, 02 state schools and 09 municipal schools, making a total of 65 visually impaired participants . Prevailed highest percentages of non-participation in AF by these individuals, and the activities carried out were mostly in physical education classes. We conclude that there is little education institutions frequented by people visually impaired, as well as insufficient service in the area of physical activity in this population.
\end{abstract}

Key-Words: People visually impaired; Physical activity; Education institutions.

Conexões: revista da Faculdade de Educação Física da UNICAMP, Campinas, v. 13, n. 4, p. 33-48, 2015. ISSN: 1983-9030. 


\section{UN DIAGNÓSTICO EN LA PRÁCTICA DE ACTIVIDADES FÍSICAS PARA PERSONAS CON DISCAPACIDAD VISUAL}

\section{RESUMEN}

El objetivo de este estudio fue verificar las Instituciones Especializadas de Educación, las escuelas públicas Asociaciones regulares y la ciudad de Santa Maria/RS que tienen los estudiantes con impedimentos visuales y diagnosticar la actividad física en esta población. Para la recolección de datos se utilizó un archivo de información. Era 01 Asociación, 02 escuelas públicas y 09 escuelas públicas, haciendo un total de 65 participantes con discapacidad visual. Prevaleció porcentajes más altos de la no participación en la FA por estos individuos, y las actividades llevadas a cabo fueron en su mayoría en las clases de educación física. Llegamos a la conclusión de que hay instituciones de educación poco atendidos por personas con discapacidad visual y la atención insuficiente en el ámbito de la actividad física en esta población.

Palabras-Clave: Personas discapacidad visual; Actividad física; Instituciones de educación. 


\section{INTRODUÇÃO}

A atividade física tem sido apontada como um componente importante para um estilo de vida saudável. ${ }^{1}$ Sabe-se que a atividade física é caracterizada como qualquer movimento corporal produzido pela musculatura esquelética, que resulta em gasto energético acima dos níveis de repouso, sendo categorizada em atividades físicas de lazer, esportivas, ocupacionais, no trabalho, dentre outras, podendo ser exemplificados por jogos, danças, esportes, exercícios físicos, lutas, deslocamentos, dentre outros. ${ }^{2}$

Ao que se refere a prática de atividades físicas por pessoas com deficiência, observa-se um maior interesse por esta população em atividades esportivas, assim como maior envolvimento e autonomia ao que se refere a atividades de lazer, trabalho e locomoção. ${ }^{3}$

Na literatura é unânime o efeito benéfico da prática de exercícios físicos nos níveis de aptidão física relacionada à saúde, na redução da taxa de mortalidade, na prevenção de doenças crônicas como hipertensão, doenças coronarianas, diabetes, entre outras, além de estar associado a melhores níveis de qualidade de vida. ${ }^{4-8}$

No entanto, pesquisas vem demonstrando que os níveis de atividade física de pessoas com deficiência visual não são satisfatórios. ${ }^{9-10}$ Além disso, grande maioria possui níveis de aptidão física precários e fortes tendências ao sedentarismo. ${ }^{11-12}$

São diversos os fatores que dificultam à prática de atividades físicas por estas pessoas, tais como, barreiras arquitetônicas, falta de capacitação de profissionais, falta de informação às pessoas com deficiência e seus familiares, etc. Em pesquisa de Scherer e Lopes ${ }^{13}$ verificouse que nos últimos 15 anos foram realizados poucos estudos envolvendo a prática de atividades físicas por pessoas adultas com deficiência visual.

Desta forma, percebe-se a necessidade da realização de diagnósticos, a fim de verificar o número de pessoas com deficiência visual, suas características e qual o seu envolvimento em práticas de atividades físicas nas mais diversas Instituições de Ensino, pois desta forma,

Conexões: revista da Faculdade de Educação Física da UNICAMP, Campinas, v. 13, n. 4, p. 33-48, out./dez. 2015. ISSN: 1983-9030. 
faz-se possível direcionar estas pessoas ao envolvimento de programas de atividades físicas, assim como, aprimorar os já existentes.

Sendo assim, o presente estudo teve como objetivo verificar as Instituições Especializadas de Ensino, Escolas Públicas Regulares e Associações da cidade de Santa Maria, RS, que possuem pessoas com deficiência visual e diagnosticar a prática de atividade física por esta população em diferentes faixas etárias.

\section{METODOLOGIA}

Primeiramente foi diagnosticado, através da $8^{\mathrm{a}}$ Coordenadoria Regional de Educação do Rio Grande do Sul e da Secretaria Municipal de Educação, as Escolas Públicas Regulares da rede estadual e municipal de Santa Maria/RS que possuíam pessoas com deficiência visual matriculados. Posteriormente, foi entrado em contato com as Instituições para verificar aquelas que possuíam alunos com deficiência visual.

Das 20 Instituições da cidade, fizeram parte do estudo 01 Associação de Cegos e Deficientes Visuais, 02 Escolas Estaduais (EE) e 09 Escolas Municipais (EM) da cidade, totalizando 12 Instituições. As demais não participaram, pois os alunos com deficiência visual não frequentavam mais os estabelecimentos.

Para a coleta de dados foi utilizada uma Ficha de Informações para Diagnóstico da Prática de Atividades Físicas por Pessoas com Deficiência Visual, baseada em Lehnhard et al. ${ }^{14}$ Esta Ficha tinha por objetivo verificar o número total de alunos com deficiência visual matriculados nas Instituições Especializadas de Ensino, Escolas Públicas Regulares ou Associações, bem como identificar as faixas etárias, série e/ou grupo, tipo e causa da deficiência visual e a participação ou não em atividades físicas nestes locais.

As Fichas foram respondidas pelas Educadoras Especiais, em virtude destas possuírem contato com os alunos desde o momento em que os mesmos inseriam-se no ambiente escolar, tendo assim um melhor acompanhamento de suas rotinas de atividades dentro e fora da Escola. As profissionais possuíam graduação em Educação Especial e 
especialização na área, trabalhando de maneira interdisciplinar com a Educação Física e demais disciplinas.

Na visita às Escolas e Associação, foi apresentado o Termo de Consentimento Livre e Esclarecido aos diretores e/ou coordenadores, para que os mesmos autorizassem a realização do estudo. O presente estudo foi aprovado pelo Comitê de Ética em Pesquisa com Seres Humanos da Universidade Federal de Santa Maria, reconhecida pela Comissão Nacional de Ética em Pesquisa (CONEF/MS), sob o protocolo n. ${ }^{\circ}$ 080.0.243.000-11, CAEE.

\section{RESULTADOS}

De acordo com os dados analisados, das Instituições que participaram do Estudo haviam 35 alunos com deficiência visual nas Associações $(n=02)$, sete nas Escolas Estaduais $(n=02)$ e 23 nas Escolas Municipais $(n=09)$. Na Tabela 1, são apresentadas as faixas etárias, os níveis de ensino, o tipo e causas da deficiência visual e outras deficiências associadas das pessoas com deficiência visual matriculadas nas Escolas e vinculadas à Associação. 
Tabela 1 - Identificação dos alunos com deficiência visual na Associação e Escolas

\begin{tabular}{|c|c|c|c|c|c|c|}
\hline \multirow[t]{2}{*}{ Alunos com DV } & \multicolumn{2}{|c|}{ Associação } & \multicolumn{2}{|c|}{$\begin{array}{c}\text { Escolas } \\
\text { Estaduais }\end{array}$} & \multicolumn{2}{|c|}{$\begin{array}{c}\text { Escolas } \\
\text { Municipais }\end{array}$} \\
\hline & $\mathbf{n}$ & $\%$ & $\mathbf{n}$ & $\%$ & $\mathbf{n}$ & $\%$ \\
\hline \multicolumn{7}{|l|}{ Faixa Etária } \\
\hline Primeira Infância (0-2 anos) & 2 & 5,7 & - & - & - & - \\
\hline Infância (2-10 anos) & 12 & 34,3 & 1 & 14,3 & 13 & 56,2 \\
\hline Adolescência (10-20 anos) & 2 & 5,7 & 6 & 85,7 & 10 & 43,5 \\
\hline Adulto (20-40 anos) & 8 & 22,8 & - & - & - & - \\
\hline Meia Idade (40-60 anos) & 9 & 25,7 & - & - & - & - \\
\hline Terceira Idade (60 anos ou mais) & 2 & 5,7 & - & - & - & - \\
\hline \multicolumn{7}{|l|}{ Níveis de Ensino } \\
\hline Educação Infantil & - & - & - & - & 4 & 17,4 \\
\hline Anos Iniciais do Ensino Fundamental & - & - & 4 & 57,1 & 12 & 52,2 \\
\hline Anos Finais do Ensino Fundamental & - & - & 1 & 14,3 & 6 & 26,1 \\
\hline Ensino Médio & - & - & 2 & 28,6 & - & - \\
\hline EJA & - & - & - & - & 1 & 4,3 \\
\hline Oficinas & 24 & 68,6 & - & - & - & - \\
\hline Nenhum & 11 & 31,4 & - & - & - & - \\
\hline \multicolumn{7}{|l|}{ Tipo da Deficiência } \\
\hline Baixa Visão & 17 & 48,6 & 7 & 100 & 16 & 69,6 \\
\hline Cegueira & 18 & 51,4 & - & - & 7 & 30,4 \\
\hline \multicolumn{7}{|l|}{ Outras Deficiências Associadas } \\
\hline Deficiência Intelectual & - & - & 5 & 71,4 & & - \\
\hline Deficiência Auditiva & - & - & 1 & 14,3 & 1 & 4,3 \\
\hline Múltiplas Deficiências & - & - & 1 & 14,3 & 4 & 17,4 \\
\hline Sem outras & 35 & 100 & - & & 18 & 78,3 \\
\hline \multicolumn{7}{|l|}{ Causas } \\
\hline Acidentes & 1 & 2,8 & - & - & 1 & 4,3 \\
\hline DSTs e/ou drogas & - & - & 5 & 71,4 & 2 & 8,7 \\
\hline Doenças ou anomalias genéticas & 5 & 14,3 & - & - & 5 & 21,7 \\
\hline Lesões cerebrais & 1 & 2,8 & - & - & 2 & 8,7 \\
\hline Retinopatias & 24 & 68,6 & - & - & 5 & 21,7 \\
\hline Desconhecida & 4 & 11,4 & 2 & 28,6 & 8 & 34,8 \\
\hline Totais & 35 & 100 & 7 & 100 & 23 & 100 \\
\hline
\end{tabular}

Pode-se identificar que as Associações atendem todas as faixas etárias, tendo uma maior participação de alunos na infância (n=12), e menor atendimento para a primeira infância $(n=02)$, adolescência $(n=02)$ e terceira idade $(n=02)$. Nas EE foi maior a inclusão de alunos na faixa etária da adolescência $(n=06)$, enquanto que na EM a faixa etária da infância $(n=13)$. No que se refere aos níveis de ensino, na Associação 24 alunos participam de oficinas, nas EE e EM a maioria dos alunos com deficiência visual estavam matriculados nos anos iniciais do ensino fundamental, $57,1 \%(n=4)$ e 52,2(n=12), respectivamente.

Conexões: revista da Faculdade de Educação Física da UNICAMP, Campinas, v. 13, n. 4, p. 33-48, out./dez. 2015. ISSN: 1983-9030. 
Quanto ao tipo de deficiência, nas Associações havia um aluno a mais com cegueira $(n=18)$ comparado aos com baixa visão $(n=17)$, enquanto que nas EE e EM havia um número maior de alunos com baixa visão ( $n=07$ e $n=16$, respectivamente). Foi verificado que dentre os alunos a deficiência intelectual foi mais associada à deficiência visual, representando um total de cinco alunos incluídos nas EE, no entanto, 53 não apresentavam outras deficiências associadas.

Dentre as causas da deficiência visual a maior foi por retinopatias $(n=24)$ daqueles alunos participantes das Associações, nas EE, o relato é de que a deficiência visual de cinco alunos se deu em virtude de DSTs e/ou drogas e nas EM por causas desconhecidas ( $n=08)$.

Demais dados não informados na Tabela 1 são de informações das Associações dos quais 35 alunos frequentadores das oficinas, nove possuíam idade para frequentar Escolas Regulares, porém três não estavam matriculados em nenhuma Instituição de Ensino Regular Público.

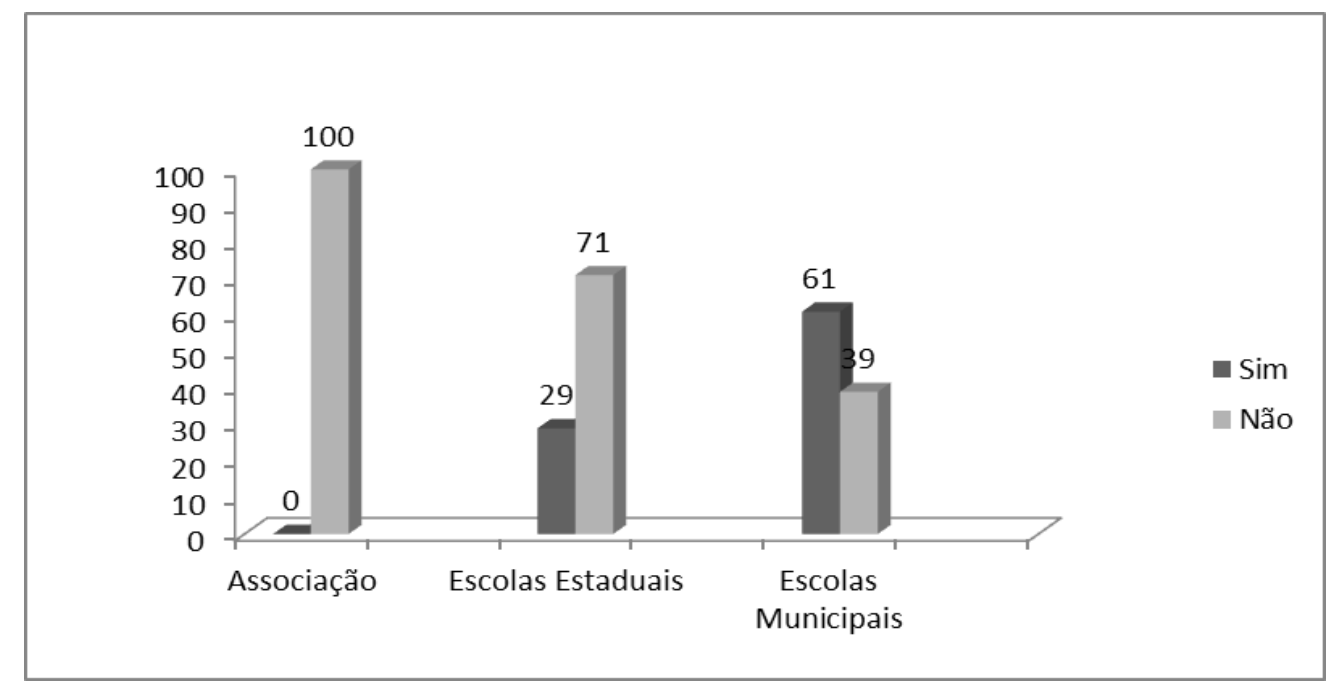

Figura 1: Prática de Atividades Físicas pelas pessoas com deficiência visual.

Pode-se visualizar que nenhum dos alunos da Associação praticava atividades físicas. Nas EE o percentual de participação em atividades físicas foi de $29 \%$ (n=02), e os que não realizavam atividades físicas somaram $71 \%(n=05)$, enquanto que nas EM o percentual de praticantes foi de $61 \%(n=14)$ e o de não praticantes de atividades físicas foi de $39 \%$ $(n=09)$.

Conexões: revista da Faculdade de Educação Física da UNICAMP, Campinas, v. 13, n. 4, p. 33-48, out./dez. 2015. ISSN: 1983-9030. 
Dentre as atividades físicas realizadas pelos alunos nas Escolas foram citadas pelas Educadoras Especiais atividades recreativas ministradas por pedagogas e participação nas aulas de Educação Física (EF).

A Tabela 2 demonstra o percentual da realização de atividades físicas em aulas de EF e atividades recreativas pelos alunos com deficiência visual das EE e EM que praticavam atividades físicas na escola.

Tabela 2 - Atividades físicas praticadas pelos alunos com deficiência visual

\begin{tabular}{lcrcrrr}
\hline INSTITUIÇÕES & \multicolumn{7}{c}{ ATIVIDADES FíSICAS PRATICADAS } & & \\
\hline & Educação Física & Atividades Recreativas & \multicolumn{2}{c}{ Total } \\
\hline & $\mathrm{n}$ & $\%$ & $\mathrm{n}$ & $\%$ & $\mathrm{n}$ & $\%$ \\
Escolas Estaduais & 2 & 100 & - & - & 2 & 100 \\
Escolas Municipais & 6 & 42,8 & 8 & 57,1 & 14 & 100 \\
\hline
\end{tabular}

Pode-se visualizar que nas EE 100\%(n=2) dos alunos com deficiência visual realizavam as atividades físicas em aulas de EF. Já nas EM, 57,1\%(n=8) dos alunos as praticavam através de atividades recreativas ministradas por pedagogas.

Quanto aos alunos que não praticavam atividades físicas, foram citados pelas Educadoras Especiais alguns motivos para a inatividade física. O Quadro 1 demonstra os motivos para a inatividade física na Associação e Escolas com seus respectivos percentuais.

Quadro 1 - Motivos para a inatividade física dos alunos na Associação e Escolas

\begin{tabular}{lrrrrrr}
\hline Motivos para a inatividade & \multicolumn{1}{c}{ Associação } & \multicolumn{2}{c}{ Escolas } & \multicolumn{3}{c}{ Escolas } \\
\hline Bullying & $\mathrm{n}$ & $\%$ & $\mathrm{n}$ & $\%$ & $\mathrm{n}$ & $\%$ \\
EF no turno inverso & 0 & 0 & 3 & 60 & 0 & 0 \\
Falta de espaço físico & 0 & 0 & 0 & 0 & 1 & 11,1 \\
Falta de Professor de EF & 35 & 100 & & 0 & 0 & 0 \\
Deficiência & 0 & 0 & 2 & 40 & 4 & 44,4 \\
Superproteção & 0 & 0 & 0 & 0 & 3 & 33,3 \\
Total & 0 & 0 & 0 & 0 & 1 & 11,1 \\
\hline
\end{tabular}

Conexões: revista da Faculdade de Educação Física da UNICAMP, Campinas, v. 13, n. 4, p. 33-48, out./dez. 2015. ISSN: 1983-9030. 
Pode-se visualizar que na Associação 100\% $(n=35)$ dos indivíduos não praticavam atividades físicas em função da falta de espaço físico. Nas EE, $60 \%(\mathrm{n}=3)$ não praticavam atividades físicas em função de sofrerem Bullying e, 44,4\% ( $\mathrm{n}=4)$ dos alunos com deficiência visual das EE não praticavam atividades físicas pela falta de docentes de EF nos Anos Iniciais do Ensino Fundamental.

Tanto na Associação como nas EE foi exposto pelas Educadoras Especiais que quando havia projetos de extensão desenvolvidos por acadêmicos de Cursos de Graduação de Educação Física das Universidades do município, que visavam a prática de atividades físicas por alunos com deficiência visual, todos participavam das atividades.

\section{DISCUSSÃO}

Os resultados do presente estudo relevaram o número reduzido de Associações e Escolas que tem a inclusão de alunos com deficiência visual. No entanto, a Associação foi o espaço em que houve uma maior inclusão, estando em sua maioria a faixa etária da infância e meia-idade. Nas Escolas Regulares a inclusão foi maior na educação infantil e ensino fundamental, sendo a faixa etária da infância e adolescência a maior participação. A prática de atividades físicas somente foi verificada nos alunos com deficiência incluídos nas escolas e na Associação os participantes não realizavam nenhum tipo de atividade.

Mesmo diante do movimento de inclusão no ensino regular ainda é reduzida a matrícula de alunos com deficiência visual. Porém, estes dados não podem ser generalizados para outros tipos de deficiência, já que o presente estudo apresentou um recorte específico da realidade da inclusão de alunos com deficiência visual nos espaços de ensino e formação.

O tipo de deficiência visual identificado entre os alunos incluídos foi em sua maioria de baixa visão, enquanto que na Associação foi maior a identificação de cegueira. Estes dados vão ao encontro a estudos populacionais, na qual indicam a baixa prevalência de cegueira em crianças e prevalência três vezes maior da baixa visão nesta faixa etária. ${ }^{20}$

Conexões: revista da Faculdade de Educação Física da UNICAMP, Campinas, v. 13, n. 4, p. 33-48, out./dez. 2015. ISSN: 1983-9030. 
No Brasil, as causas da deficiência visual são em decorrência de distúrbios da retina, incluindo retinopatias, retinopatia diabética e glaucoma. ${ }^{21}$ Assim como em estudo de Brito e Veitzman ${ }^{20}$ há percentuais relativos a causas desconhecidas, porém sem justificativas para tais resultados. Vale salientar que o conhecimento da origem das deficiências se faz importante, uma vez que a determinação das causas pode fornecer suporte e informações para que sejam feitas ações objetivando a prevenção e promoção da saúde ocular, assim como implementação do tratamento. ${ }^{20,} 22$ Já o grande número de alunos nas EE com deficiência intelectual associada, possivelmente deve-se ao fato da deficiência ter sido causada pela exposição a doenças sexualmente transmissíveis (DST) em período gestacional da mãe. ${ }^{23}$

A diferença entre os tipos de causas da deficiência pode ser barreiras para a não participação em atividades físicas, já que diferentes estratégias pedagógicas devem ser adotadas. ${ }^{11-12}$ Nas falas das Educadoras Especiais as barreiras vão desde os espaços físicos, quanto o preconceito, falta de professor e a própria limitação da deficiência. Possivelmente isso deve-se ao despreparo na gestão escolar, espacial e organizacional, tanto na atuação pedagógica, quanto das relações sociais para a inclusão. ${ }^{15} \mathrm{~A}$ formação profissional também esteve presente em estudos que trataram sobre a inclusão de alunos com deficiência visual no ensino regular. ${ }^{17}$ Demais estudos também evidenciaram barreiras para a participação em atividades físicas como, dificuldades de transporte e barreiras arquitetônicas. ${ }^{27}$ As barreiras sociais na relação afetiva com seus pares e professores, pode ser percebido nas situações de bullying, ou até mesmo o medo de exposição e exclusão como visto em outros estudos ${ }^{27}$, 30

No entanto, a reduzida participação em atividades físicas dos alunos investigados poderá ter implicações tanto nos aspectos sociais, afetivos, como também motores desde a infância. ${ }^{26}$ Nesta fase de crescimento o estímulo precoce é uma intervenção importante para a aquisição de habilidades e capacidades motoras necessárias para uma maior autonomia e independência funcional. ${ }^{12,}$ 27-28, 33-34 A participação em aulas de Educação Física e atividades físicas são fundamentais por possibilitar variadas experiências de movimento e socialização em todas as faixas etárias do ciclo da vida. ${ }^{35}$ Enquanto 
componente curricular na Escola a Educação Física pode ser facilmente um espaço de inclusão, interação e troca de experiências. ${ }^{30}$

Kasper et al. ${ }^{18}$ afirmam que houve um aumento de matrículas de alunos com deficiência nas Escolas Públicas Regulares, porém o número evasão pela falta de condições adequadas à sua permanência, ainda é elevado. Isto pode ser verificado no presente estudo em relação aos alunos em idade escolar, mas que apenas participavam da Associação. A participação em oficinas de capacitação e formação se faz importante na medida em que colaboram ao desenvolvimento psicomotor e no auxílio à inclusão de qualidade nas Escolas, porém, não devem substituir de forma alguma a educação escolar em qualquer nível. ${ }^{19}$

Diante os dados do estudo, a inclusão de alunos com deficiência nos mais diversos contextos de ensino e formação devem ser articulados com perspectivas, atitudes e ações que promovam as oportunidades de vivência entre todos os envolvidos. Esta troca irá favorecer não somente aos alunos com deficiência visual, mas contribuirá para melhorar as relações sociais e afetivas entre os alunos, para tornar um ambiente mais positivo e agradável, reduzindo os agravos a saúde emocional e física.

\section{CONCLUSÃO}

Pode-se verificar que são poucas as Instituições de Ensino, no município de Santa Maria/RS, frequentadas por alunos com deficiência visual e este cenário é ainda mais preocupante quando em relação a prática de atividades físicas, pois quanto maior a idade menor o engajamento em atividades físicas.

As barreiras identificadas das Escolas para a pouca participação vão desde os espaços físicos, quanto barreiras sociais e da própria deficiência enquanto limitação. Sendo assim, faz-se necessário direcionar ações voltadas a inclusão efetiva de alunos com deficiência visual tanto nos espaços escolares, quanto nas Associações, para que seja oportunizado o acesso as mais variadas práticas de atividades físicas, de forma a contribuir com um estilo de vida mais ativo.

Conexões: revista da Faculdade de Educação Física da UNICAMP, Campinas, v. 13, n. 4, p. 33-48, out./dez. 2015. ISSN: 1983-9030. 


\section{REFERÊNCIAS}

${ }^{1}$ AMERICAN COLLEGE OF SPORTS MEDICINE (ACSM). Diretrizes do ACSM para os testes de esforço e sua prescrição. 8. ed. Rio de Janeiro: Guanabara Koogan, 2010. p. 272

${ }^{2}$ CASPERSEN, C. J.; POWELL, K. E.; CHRISTENSON, G. M. Physical activity, exercise, and physical fitness: definitions and distinctions for health-related research. Public Health Reports, Rockville, v. 100, n. 2, p. 126-131, 1985.

${ }^{3}$ LIMA, C. R. F.; GORGATTI, M. G.; DUTRA, M. C. A influência do esporte na qualidade de vida das pessoas com deficiência visual. Revista Brasileira de Ciências da Saúde, São Caetano do Sul, ano 8, n. 23, p. 40-47, jan./mar. 2010.

${ }^{4}$ WORLD HEALTH ORGANIZATION (WHO). Global recommendations on physical activity for health. Geneva, 2010.

${ }^{5}$ PAFFENBARGER JÚNIOR, R. S.; LEE, I. M. Physical activity and fitness for health and longevity. Research Quarterly for Exercise \& Sport, Reston, v. 67, p.11-28, 1996.

${ }^{6}$ NAHAS, M. V. Atividade física, saúde e qualidade de vida: conceitos e sugestões para um estilo de vida ativo. 6. ed. Londrina: Midiograf, 2013. p. 335

${ }^{7}$ HÄKKINEN, A. et al. Association of physical fitness with health-related quality of life in Finnish young men. Health and Quality of Life Outcomes, London, v. 8, n. 15, p. 1-8, 2010.

${ }^{8}$ PICCOLI, J. C. J.; OLIVEIRA, G. T.; FERRAREZE, M. E. A prática de atividade física na região do Vale dos Sinos no Estado do Rio Grande do Sul. Revista Brasileira de Ciência e Movimento, Brasília, v. 18, n. 1, p. 42-47, 2010. 
${ }^{9}$ INTERDONATO, G. C.; GREGUOL, M. Qualidade de vida e prática habitual de atividade física em adolescentes com deficiência. Revista Brasileira de Crescimento e Desenvolvimento Humano, São Paulo, v. 21, n. 2, p. 282-295, 2011.

${ }^{10}$ ASLAN, U. B.; CALIK, B. B.; KITIS, A. The effect of gender and level of vision on the physical activity level of children and adolescents with visual impairment. Research in Developmental Disabilities, Elmsford, v. 33, n. 6, p. 1799-1804, jun. 2012.

${ }^{11}$ GORGATTI, M. G.; TEIXEIRA, L.; VANÍCOLA, M. C. Deficiência visual. In: TEIXEIRA, L. (Org.). Atividade física adaptada e saúde: da teoria à prática. São Paulo: Phorte, 2008. p. 399-412.

${ }^{12}$ GREGUOL, M.; ROSE JÚNIOR, D. Aptidão física relacionada à saúde de jovens cegos em escolas regulares e especiais. Revista Brasileira de Crescimento e Desenvolvimento Humano, São Paulo, v. 19, n. 1, p. 42-53, 2009.

${ }^{13}$ SCHERER, R. L.; LOPES, A. S. Atividade física habitual em adultos com deficiência visual: uma revisão sistemática. Pensar a Prática, Goiânia, v. 16, n. 1, p. 1-319, jan./mar. 2013.

${ }^{14}$ LEHNHARD, G. R. et al. A inclusão de alunos com deficiência em escolas públicas e em aulas de Educação Física: um diagnóstico. EDFeportes.com: revista digital, Buenos Aires, ano. 14, n. 139, jan. 2009. <http://www.efdeportes.com/efd139/inclusao-de-alunos-comdeficiencia-em-educacao-fisica.htm>

Acesso em: 20 dez. 2014.

${ }^{15}$ FERREIRA, W. B. Educação inclusiva: será que sou a favor ou contra uma escola de qualidade para todos? Inclusão: revista de educação especial, Brasília, ano 1, n. 1, p. 40 46, out. 2006.

Conexões: revista da Faculdade de Educação Física da UNICAMP, Campinas, v. 13, n. 4, p. 33-48, out./dez. 2015. ISSN: 1983-9030. 
${ }^{16}$ DUTRA, R. S.; SILVA, S. S. M.; ROCHA, R. C. S. A educação inclusiva como projeto da escola: O lugar da educação física. Revista Adapta, Rio Claro, ano 2, n. 1, p. 7-12, 2006.

${ }^{17}$ COSTA, V. B. Inclusão escolar na educação física: reflexões acerca da formação docente. Motriz: revista de educação física, Rio Claro, v. 16 n. 4 p. 889-899, out./dez. 2010.

${ }^{18}$ KASPER, A. A.; LOCH, M. V. P.; PEREIRA, V. L. D. V. Alunos com deficiência matriculados em escolas públicas de nível fundamental: algumas considerações. Educar, Curitiba, n. 31, p. 231-243, 2008.

${ }^{19}$ BRASIL. Procuradoria Federal dos Direitos do Cidadão. O acesso de alunos com deficiência as escolas e classes comuns da rede regular. 2. ed. Brasília, 2004.

${ }^{20}$ BRITO, P. R.; S, VEITZMAN. S. Causas de cegueira e baixa visão em crianças. Arquivos Brasileiros de Oftalmologia, São Paulo, v. 63, n. 1, p. 49-54, fev. 2000.

${ }^{21}$ SALOMÃO, S. R.; MITSUHIRO, M. R. K. H.; BELFORT JÚNIOR, R. Visual impairment and blindness: an overview of prevalence and causes in Brazil. Anais da Academia Brasileira de Ciências, v. 81, n. 3, p. 539-549, 2009.

${ }^{22}$ CASTRO, S. S. et al. Deficiência visual, auditiva e física: prevalência e fatores associados em estudo de base populacional. Caderno de Saúde Pública, Rio de Janeiro, v. 24, n. 8, p. 1773-1782, ago. 2008.

${ }^{23} \mathrm{GIR}$, E. et al. Medidas preventivas contra a AIDS e outras doenças sexualmente transmissíveis conhecidas por universitários da área de saúde. Revista Latino Americana de Enfermagem, v. 7, n.1, p. 11-17, 1999.

Conexões: revista da Faculdade de Educação Física da UNICAMP, Campinas, v. 13, n. 4, p. 33-48, out./dez. 2015. ISSN: 1983-9030. 
${ }^{24}$ FILUS, J. F.; MARTINS JÚNIOR, J. Reflexões sobre a formação em educação física e a sua aplicação no trabalho junto às pessoas com deficiência. Revista da Educação Física/UEM, Maringá, v. 15, n. 2, p. 79-87, 2004.

${ }^{25}$ GORGATTI, M. G. Educação física escolar e inclusão: uma análise a partir do desenvolvimento motor e social de adolescentes com deficiência visual e das atitudes dos professores. 2005. Tese (Doutorado em Educação Física) - Universidade de São Paulo, São Paulo, 2005.

${ }^{26}$ SILVA, E. L.; RODRIGUES, G. M. O Acesso ao esporte para crianças e adolescentes com deficiência: da acessibilidade aos programas públicos. In: CONGRESSO BRASILEIRO DE CIÊNCIAS DO ESPORTE, 15.; CONGRESSO INTERNACIONAL DE CIÊNCIAS DO ESPORTE, 3., Salvador, 2009. Anais... Salvador: CBCE, 2009. p. 112.

${ }^{27}$ MOREIRA, W. C. et al. Fatores que influenciam a adesão de deficientes motores e deficientes visuais a prática esportiva. EDFeportes.com: revista digital, Buenos Aires, ano 11, n. 104, jan. 2007.

$<$ http://www.efdeportes.com/efd104/deficientes-motores-visuais.htm>

Acesso em: 20 nov. 2014.

${ }^{28}$ SOUZA, R. P.; CAMPOS, D. Atividades esportivas para deficientes visuais: uma análise quantitativa do município do Rio de Janeiro. Revista de Educação Física, Rio de Janeiro, n. 142, p. 50-57, set. 2008.

${ }^{29}$ BRASIL. Ministério dos Esportes. Secretaria Especial dos Direitos Humanos Coordenadoria Nacional para Integração da Pessoa Portadora de Deficiência. Acessibilidade. Brasília, 2005. 
${ }^{30}$ ALVES, M. L. T.; DUARTE, E. A inclusão do deficiente visual nas aulas de educação física escolar: impedimentos e oportunidades. Revista da Educação Física/UEM, Maringá, v. 27, n. 2, p. 231-237, 2005.

${ }^{31}$ TUBINO, M. J. G. Dimensões sociais do esporte. 2. ed. São Paulo: Cortez, 2001. p. 95.

${ }^{32}$ ARAÚJO, A. V. A inclusão por meio do esporte: o ponto de vista da criança. 2006. Monografia (Especialização em Esporte Escolar) - Centro de Educação a Distância, Universidade de Brasília, Fortaleza, 2006.

${ }^{33}$ WINNICK, J. P. Educação física e esportes adaptados. Barueri: Manole, 2004. p. 552.

${ }^{34}$ NEVES, G. N.; FRASSON, A. C.; CANTORANI, J. R. H. Educação física adaptada ao deficiente visual. 2009 . Disponível em:

$<$ http://www.educadores.diaadia.pr.gov.br/modules/mydownloads_01/singlefile.php?cid=3 6\&lid $=3439>$.

Acesso em: 02 set. 2014.

${ }^{35}$ GALLAHUE, D. L.; OZMUN, J. C. Compreendendo o desenvolvimento motor: bebês, crianças, adolescentes e adultos. São Paulo: Phorte, 2001. p. 487.

Recebido em: 09 abr. 2015

Aceito em: 13 nov. 2015

Contato: rafaellarighes@hotmail.com

Conexões: revista da Faculdade de Educação Física da UNICAMP, Campinas, v. 13, n. 4, p. 33-48, out./dez. 2015. ISSN: 1983-9030. 